\title{
Extravasation Injuries, Pressure Ulcers and Ocular Surface Disorders in a Tertiary Paediatric Intensive Care Unit in South India
}

\section{Puja Amatya ${ }^{1}$ and Kalaimaran Sadasivam ${ }^{2}$}

${ }^{1}$ Department of Paediatrics, Patan Academy of Health Sciences, Lalitpur, Nepal

${ }^{2}$ Department of Paediatric Critical Care unit, Kanchi Kamakoti CHILDS Trust Hospital, Chennai, India

\author{
Correspondence: \\ Dr. Puja Amatya \\ Assistant Professor \\ Department of Paediatrics \\ Patan Academy of Health Sciences \\ Lagankhel, Lalitpur, Nepal \\ Email: \\ pujaamatya@pahs.edu.np
}

DOI:10.3126/jnps.v39i1.27156

Submitted on: 2020-01-02

Accepted on: 2020-02-08

Acknowledgements: Nursing staffs, Department of Paediatric Critical Care Unit, Kanchi Kamakoti CHILDS Trust Hospital, Chennai, India

Funding: Nil

Conflict of Interest: None declared

Permission form IRB: Yes

To cite this article: Amatya $P$, Sadasivam K. Extravasation injuries, pressure ulcers and ocular surface disorders in a tertiary paediatric intensive care unit in South India. J Nepal Paediatr Soc. 2019;39(1):29-34.

\section{ABSTRACT}

Introduction: Extravasation injuries, pressure ulcers (PU), and ocular surface disorders (OSD) like exposure keratitis are common problems that we encounter in critically ill children admitted in Paediatric Intensive Care unit (PICU). There is sparse data regarding these injuries due to under reporting by staffs in intensive care unit. The primary aim of this study was to know the prevalence of extravasation injuries, ocular surface disorders and pressure ulcers in tertiary intensive care unit of south India. The secondary objective of this study was to re-evaluate the prevalence of these injuries after interventions.

Methods: During pre-intervention period, number of children with extravasation injuries, pressure ulcers and ocular surface disorders were identified. The qualitative improvement practices was implemented to decrease the prevalence of these injuries. This was followed by re-evaluation of these injuries during post-intervention period.

Results: During pre-intervention period the overall prevalence of these injuries was $36 / 273$ (13.2\%). The prevalence of extravasation injuries, pressure ulcers and ocular surface disorders was 16/273 $(5.9 \%), 12 / 273(4.4 \%)$ and $8 / 273(2.9 \%)$ respectively. The qualitative improvement practices were implemented in intensive care unit. During post-intervention period, the prevalence of these injuries was reduced to $10 / 157(6.4 \%)$. The extravasation injuries reduced to $4 / 157$ (2.5\%), PUs to $4 / 157(2.5 \%)$ and OSD to $2 / 157(1.3 \%)$.

Conclusions: The extravasation injuries, PUs and OSD are common problems in critically ill children. The qualitative improvement practices should be implemented and reinforced in intensive care units to prevent these injuries.

Key words: Extravasation injuries; Ocular surface disorder (OSD); Paediatric intensive care unit (PICU); Pressure ulcers (PUs)

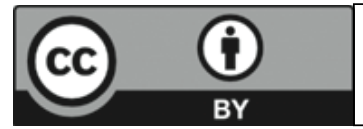

This work is licensed under creative common attribution 3.0 license 


\section{INTRODUCTION}

Extravasation injuries, pressure ulcers (PU) and ocular surface disorders (OSD) are common problems that are seen in critically ill children of Paediatric Intensive Care Unit (PICU). Extravasation is the inadvertent administration of intravenous medicines from the vein into surrounding tissue either by leakage or direct exposure caused by improper cannula placement and venepuncture techniques. ${ }^{1}$ The incidence of extravasation injuries in admitted patients is reported as $0.1 \%$ to $6.5 \%$ but the true incidence may be higher because of poor documentation and reporting. ${ }^{2-4}$ Extravasation injuries can cause pain, redness, and swelling, damage to vessels, nerves or tendon and can predispose to local and invasive infection.

Pressure ulcer is a localised injury to the skin and or underlying tissue as a result of pressure or pressure in combination with shear forces. ${ }^{5}$ Critically ill children admitted in PICU are at risk for pressure ulcers due to immature skin, decreased perfusion, decreased mobility, altered neurologic responsiveness, volume overload, moisture, and medical devices. ${ }^{6}$ The PU occurs usually at pressure dependent regions of bony prominence due to prolonged contact with a firm surface. Ocular surface disorders (OSD) have been reported to occur in up to $60 \%$ of critically ill patients. Critically ill patients in intensive care unit have impaired ocular defence mechanisms because of different associated conditions like multi-organ dysfunction syndrome, metabolic disturbances, mechanical ventilation and unconsciousness. ${ }^{7,8}$ The use of sedation and muscle relaxants inhibit contraction of the orbicularis oculi muscle that results in incomplete eyelid closure. Lagopthalmos has been reported to occur in $20 \%$ to $75 \%$ of sedated patients in intensive care units. ${ }^{7-11}$ There is drying of the eyes, desiccation of the cornea epithelial cells, corneal ulceration and increased risk of microbial keratitis due to incomplete closure of eye lids. This may lead to corneal thinning and perforation if untreated.

There is sparse data of extravasation, PU and OSD in critically ill children in our settings. This may be due to under reporting of these events in PICU. The primary aim of this study was to know the prevalence of extravasation injuries, PU and OSD in tertiary care PICU of South India. The secondary objective of this study was to re-evaluate the prevalence of these injuries after implementation of qualitative improvement interventions.

\section{METHODS}

This was a cross-sectional quality improvement study. This study was done in tertiary care paediatric critical care unit of South India. The preintervention period was from 1st of October 2018 to $31 \mathrm{st}$ December 2018. The post-intervention period was from 1st January 2019 to 30th March 2019. The prevalence of extravasation injuries, ocular surface disorders and pressure ulcers was seen during first three months. The guidelines and practices which were followed in PICU were implemented and reinforced to reduce the prevalence of these injuries. After intervention of these guidelines, again the prevalence of these injuries were seen to see whether these injuries are decreasing after intervention.

Extravasation injuries have been classified into four stages of increasing severity, which are thought to be useful in predicting injury prognosis and in determining the best treatment results. ${ }^{12}$ The four stages are:

Stage 1: a painful intravenous site, no erythema and swelling, flushes with difficulty.

Stage 2: a painful intravenous site, slight swelling, redness, no blanching, brisk capillary refill below infiltration site, good pulse volume below infiltration site.

Stage 3: a painful intravenous site, marked swelling, blanching, cool to touch, brisk capillary refill below infiltration site, good pulse volume below infiltration site.

Stage 4: a painful intravenous site, very marked swelling, blanching, cool to touch, capillary refill of $>4$ seconds, decreased or absent pulse, skin breakdown or necrosis.

Ocular surface disorders (OSD) ${ }^{13}$

1. Exposure keratopathy represents a dryness of the cornea due to incomplete lid closure.

2. Chemosis is conjuctival edema. 
3. Microbial infections: The most common isolated organisms are Pseudomonas aeruginosa, Acinetobacter spp. and Staphylococcus epidermidis.

4. Conjunctivitis: It manifests as sticky eye 5. Microbial keratitis: The damaged cornea is especially vulnerable to bacterial invasion which can occur very rapidly.

Pressure Ulcers (PU):

PUs are classified by the depth and severity. ${ }^{5}$

Stage $I$ is non-blanchable erythematous skin that may be painful, soft, and warmer or cooler than adjacent tissue.

Stage II involves partial dermal loss (e.g. shallow open ulcer or intact blister).

Stage III has dermal loss wherein subdermal elements (e.g. subcutaneous fat) are visualised.

Stage IV is full-thickness tissue loss with exposed bone, tendon, or muscle. Unstageable ulcers are full-thickness wounds covered by slough and/or eschar.

The pre-interventional and post-interventional data were analysed using Microsoft Excel 2010.

\section{RESULTS}

The overall prevalence of extravasation injuries, PU and OSD was 36/273 (13.2\%) during preintervention period. Out of 36 children, 20/36 $(55.5 \%)$ were males and $16 / 36(44.4 \%)$ were females and ratio was $1.25: 1$. These injuries were most common in < one year of age (14/36-39\%) followed by five to 10 years, one to five years and 10 to 15 years as shown in Figure 1.

The prevalence of extravasation injuries, PU and OSD was $16 / 273(5.9 \%), 12 / 273(4.4 \%)$ and $8 / 273$ $(2.9 \%)$ respectively. Out of 16 extravasation injuries, 4/16 (25\%) had stage 1, 8/16 (50\%) had stage 2, 2/16 (12.5\%) had stage 3 and 2/16 (12.55) had stage 4 classification. The extravasation injuries were more common in $<$ one year of age which was 10/16 (62.5\%). Out of 16, 11/16 (68.7\%) extravasation injuries were on upper limbs. The most common reported symptoms were swelling 13/16 (81.2\%) and pain 10/16 (62.5\%). Out of $12 \mathrm{PU}, 4 / 12$ (33.3\%) had stage I and 8/12 $(66.6 \%)$ had stage II PU. There was no stage III and

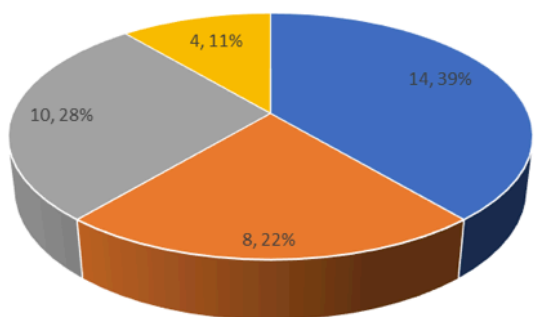

- <1year " 1-5years " 5-10years " 10-15years

Figure 1. Age distribution of extravasation injuries, pressure ulcers and ocular surface disorders $(\mathrm{N}=36)$.

IV pressure ulcers. The PU due to device was $5 / 12$ (41.6\%). The device related PUs were related to Non-invasive ventilation like nasal CPAP, BiPAP, ECG leads and pulse oximeter probe. Out of eight OSD, 5/8 (62.5\%) had exposure keratitis and 3/8 $(37.5 \%)$ had conjunctival chemosis. Nobody had conjunctivitis or purulent keratitis. OSD was most common in $6 / 8(75 \%)$ of children who who were mechanically ventilated, were on sedation and paralysis and stayed for more than one week in PICU.

The quality improvement practices was implemented as well as reinforced to PICU during the post-intervention period. For extravasation injuries, intravenous cannula as well as arterial line site was checked by nurse and on call doctor in each shift and mentioned in daily checklist. For arterial line, perfusion of limb as well as saturation of that limb was monitored hourly.

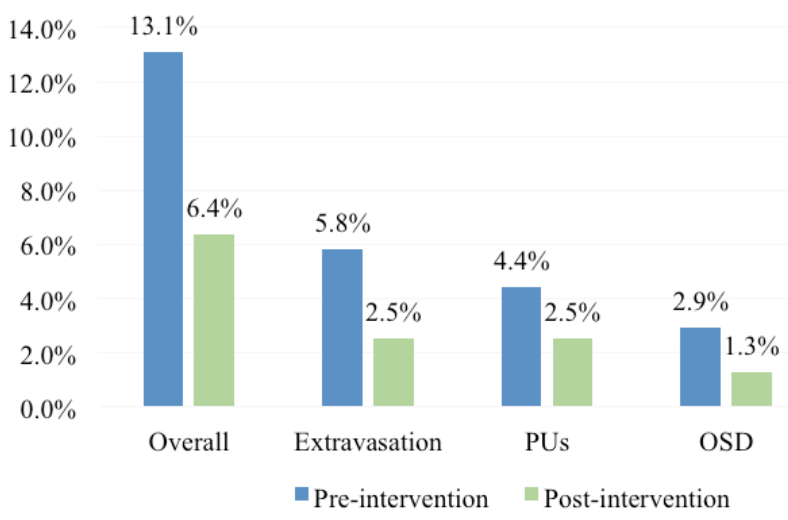

Figure 2. Prevalence of Extravasation, Pressure ulcers (PUs) and Ocular surface disorders (OSD) during Preintervention and Post-intervention period in PICU 
For ocular surface disorders, eye examination was done as a part of checklist in each shift by nurse and doctor. In mechanically ventilated children, artificial tear eye drops was kept hourly and lacrigel ointment was kept every six hours. Eye taping was done routinely in all mechanically ventilated children and this practice was reinforced in unit. The ophthalmological evaluation was done if any signs of OSD was identified and managed according to the ophthalmologist plan.

For pressure ulcers, position was changed every two hours for mechanically ventilated children as well as for those who had muscle weakness or paralysis. Daily assessment of skin for pressure ulcers was done in each shift by nurse and doctor and it was mentioned in a checklist. To avoid PUs, NIMBUS bed was used in children who required mechanical ventilation for more than one week as well as on children with muscle weakness and paralysis. The routine use of coconut oil to prevent dryness in PICU was reinforced. For the pressure ulcers related to device, examination of skin around and beneath the device was done in every shift by nurse and doctor and was mentioned in checklist. The routine use of duoderm for application of mask in children requiring non-invasive ventilation and invasive ventilation was reinforced.

During post-intervention period, the overall prevalence of extravasation injuries, OSD and PUs was reduced to $10 / 157(6.4 \%)$. The prevalence of extravasation injuries was reduced to $4 / 157(2.5 \%)$. Out of four extravasation injuries three had stage 1 and one had stage 2 injuries. The prevalence of PUs was reduced to $4 / 157$ (2.5\%). Out of four PUs, three had stage I and one had stage II pressure ulcer. The device related pressure ulcer was reduced to one. The prevalence of OSD was reduced to $2 / 157(1.3 \%)$. Out of two OSD, one had exposure keratitis and one had conjunctival chemosis. The prevalence of extravasation injuries, PUs and OSD during pre-intervention and postintervention is summarised in Figure 2.

\section{DISCUSSION}

Extravasation injuries are more common in paediatric population due to the fragility of vessels. There is a lack of literature about extravasation injuries in the paediatric population. Extravasation injuries are caused by accidental leakages of medications that are given intravenously. They can cause complications in form of short-term pain and longer-term scarring. These injuries may be severe in small children due to their fragile veins and skin. Children in PICU are at increased risk of ocular surface disorders due to poor eyelid closure, decreased blink reflex, and increased exposure to pathogenic micro-organisms. PUs are also common in PICU especially in bedridden patients.

The overall prevalence of extravasation injuries, OSD and PUs was $13.2 \%$. The prevalence of extravasation injuries was $5.9 \%$ which was higher compared to Ya-Min Yan et al. ${ }^{14}$ where its prevalence was only $1.79 \%$. According to Vanessa et $\mathrm{a} \mathrm{l}^{15}$ extravasation injuries was more common in infants (40\%) which was comparable to this study (39\%). Previous studies ${ }^{16,17}$ have identified that limbs are more prone for extravasation injuries with up to $60 \%$ occurring in upper limbs which was a similar to our study (62.5\%). According to Ghanem et $\mathrm{al}^{17}$ almost $60 \%$ of extravasation injuries in children occurred in less than one year of age which was higher than our study where it was (43.7\%). The reason for this could be upper limb preference for cannulation as well as cannula displacement is more common in upper limbs due to excessive movements.

In this study the prevalence of extravasation injuries was 16/273 (5.9\%) which was higher compared to previous studies where they reported results between $0.03 \%-1.79 \% .^{18-21}$ Out of 16 extravasation injuries, 4/16 (25\%) had stage one, $8 / 16(50 \%)$ had stage 2, $2 / 16(12.5 \%)$ had stage three and 2/16 (12.5\%) had stage four classification which was higher compared to the study by Ya-Min et al. ${ }^{14}$ where grade $1,2,3,4$ were $22.2 \%, 44.4 \%$, $27.8 \%$ and $5.6 \%$ respectively. The most common reported symptoms were swelling 13/16 (81.2\%) and pain $10 / 16(62.5 \%)$ which was comparable to the study by Ya-Min et al. ${ }^{14}$ where swelling was $94.44 \%$ and pain was $61.11 \%$.

In our study OSD comprises $8 / 273$ (2.9\%) which is much less compared to the studies 7,8 which have been reported to occur in up to $60 \%$ of critically ill patients. Out of eight OSD, 5/8 (62.5\%) had 
exposure keratitis and 3/8 (37.5\%) had chemosis. Nobody had conjunctivitis or purulent keratitis. In our study OSD was found in eight patients who stayed in PICU for more than seven days and who was mechanically ventilated and was on sedation and paralysis which was similar to the findings by the study of Imanaka et al. ${ }^{24}$ Out of eight patients who had sedatives or muscle relaxants administered continuously for more than 48 hours in the PICU, $5 / 8(62.5 \%)$ developed keratitis which was comparable to the study by Imanaka et al. ${ }^{22}$ where it was $60 \%$.

The prevalence of PUs was 12/273 (4.4\%). Out of 12 pressure ulcers, $4 / 12(33.3 \%)$ had stage I and $8 / 12(66.6 \%)$ had stage II PUs in PICU. Nobody had stage III or IV PUs which was more than reported by Christine et al. ${ }^{23}$ where the overall incidence of pressure ulcers was $10.2 \%$ and lower than by Anna-Barbara et al. ${ }^{24}$ (44\%). As other studies $^{24,25}$ this study also had PUs related to devices were due to use of CPAP or BIPAP which was $5 / 12(41.6 \%)$. In the study by Anna-Barbera et al. ${ }^{24}$ most patients with PUs (80\%) had category 1 ulcers where as in our study most PUs were of category $2(8 / 12-66.6 \%)$. The prevalence of category 2 PUs was $66.6 \%$ which was higher than previous reported studies by Anna et $\mathrm{al}^{24}$ where it accounts for $3 \%$ of the total PUs.
According to Marty et al. ${ }^{26}$ a leadership team approach was assembled to reduce the PU rate by $50 \%$ in the PICU and NICU. QI intervention done were frequent, thorough skin assessments, preventative interventions comprehensive education, clinical staff empowerment (e.g. skin champions), and systems change (e.g., skin rounds). After this intervention they were able to achieve the aim to reduce PUs by less than $50 \%$.

The limitation of this study was that prevalence of these injuries was seen only for three months after post-intervention. Hence, there is lacking evidence whether there was persistence decrease of these injuries after intervention.

\section{CONCLUSIONS}

Extravasation injuries, OSD and PUs are one of the serious issues in paediatric critical care unit if not treated timely. The implementation and continuous reinforcement of guidelines will definitely help to reduce these injuries in critically ill children.

\section{REFERENCES}

1. Kumar RJ, Pegg SP, Kimble RM. Management of extravasation injuries. ANZ J Surg. 2001;71(5):285-9. DOI: https://doi.org/ 10.1046/j.1440-1622.2001.02104.x

2. Camp-Sorrell D. Developing extravasation protocols and monitoring outcomes. J Intraven Nurs 1998;21(4):232-239.

3. Cartlidge PHT, Fox PE, Rutter N. The scars of newborn intensive care. Early Hum Dev. 1990;21(1):1-10. https://oi.org/ 10.1016/0378-3782(90)90105-R

4. Wilkins CE, Emmerson AJB. Extravasation injuries on regional neonatal units. Arch Dis Child Fetal Neonatal Ed. 2004;89(3): 274-5. DOI: https://doi.org/10.1136/adc.2003.028241

5. National Pressure Ulcer Advisory Panel (NPUAP) and European Pressure Ulcer Advisory Panel (EPUAP), 2009. Prevention and treatment of pressure ulcers: clinical practice guideline. National Pressure Ulcer Advisory Panel, Washington DC. Available at: http://npuap.org.

6. Schindler CA, Mikhailov TA, Fischer K, Lukasiewicz G, Kuhn EM, Duncan L. Skin integrity in critically ill and injured children. Am J Crit Care. 2007;16(6):568-574.

7. A. Grixti, M. Sadri, J. Edgar, and A. V. Datta. Common ocular surface disorders in patients in intensive care units. The Ocular Surface. 2012;10:26-42. DOI: https://doi.org/10.1016/j.jtos.2011.10.001

8. Joyce N. Eye care for the intensive care patient; A Systematic Review. The Joanna Briggs Institute for Evidence Based Nursing and Midwifery, Adelaide, Australia, 2002. 
9. Mela EK, Drimtzias EG, Christofidou MK, Filos KS, Anastassiou ED, Gartaganis SP. Ocular surface bacterial colonisation in sedated intensive care unit patients. Anaesthesia and Intensive Care. 2010;38(1):190-3. DOI: https://doi.org/ $10.1177 / 0310057 \mathrm{X} 1003800129$

10. Kirwan JF, Potamitis T, El-Kasaby H, Hope-Ross MW, Sutton GA. Lesson of the week: microbial keratitis in intensive care. Br Med J. 1997;314(7078):433-4. DOI: https://doi.org/10.1136/bmj.314.7078.433

11. F. Mercieca, P. Suresh, A. Morton, and A. Tullo. Ocular surface disease in intensive care unit patients. Eye 1999;13(2):231-6. DOI: https://doi.org/10.1038/eye.1999.57

12. Millam DA. Managing complications of i.v. therapy (continuing education credit). Nursing. 1988;18:34-43. DOI: https://doi.org/ $10.1097 / 00152193-198803000-00020$

13. The Royal College of Ophthalmologists. Ophthalmic Services Guidance. Eye Care in the Intensive Care Unit (ICU), June $2017 ; 1-17$

14. Ya-Min Yan, Mei Gong, Jia-Ling Chen, Dan Li, Ting-Ting Xu, Huan Zou, et al. Incidence, risk factors and treatment outcomes of drug extravasation in pediatric patients in China. The Turkish Journal of Paediatrics 2017;59:162-8. DOI: https://doi.org/ 10.24953/turkjped.2017.02.008

15. Vanessa P, Rumi M, Tracie N, Pia D, Avash S, Roxane C. Describing Intravenous Extravasation in Children (DIVE Study). Can J Hosp Pharm 2011;64(5):340-5. DOI: https://doi.org/10.4212/cjhp.v64i5.1069

16. Goutos I, Cogswell LK, Giele H. Extravasation injuries: a review. J. Hand Surg. Eur 2014; 39:808-18. DOI: https://doi.org/ $10.1177 / 1753193413511921$

17. Ghanem AM, Mansour A, Exton R, Powell J, Mashhadi S, Bulstrode N, et al. Childhood extravasation injuries: improved outcome following the introduction of hospital-wide guidelines. J. Plast. Reconstr. Aesthet. Surg 2015; 68: 505-18. DOI: https:// doi.org/10.1016/j.bjps.2014.12.029

18. Sakaida E, Sekine I, Iwasawa S, Kurimoto R, Uehara T, Ooka $\mathrm{Y}$, et al. Incidence, risk factors and treatment outcomes of extravasation of cytotoxic agents in an outpatient chemotherapy clinic. Jpn J Clin Oncol 2014;44:168-71. DOI: https://doi.org/ $10.1093 / \mathrm{jjco} / \mathrm{hyt} 186$

19. Alami Z, Nasri S , Ahid S, Kacem HH. Extravasation of contrast medium during CT examination: an observational case control study. Pan Afr Med J 2015;20:89. DOI: https://doi.org/10.11604/pamj.2015.20.89.3276

20. Gilbar PJ, Carrington CV. The incidence of extravasation of vinca alkaloids supplied in syringes or mini-bags. J Oncol Pharm Pract 2006;12:113-8. DOI: https://doi.org/10.1177/1078155206070448

21. Wang CL, Cohan RH, Ellis JH, Adusumilli S, Dunnick NR. Frequency, management, and outcome of extravasation of nonionic iodinated contrast medium in 69,657 intravenous injections. Radiology 2007;243:80-87. DOI: https://doi.org/10.1148/radiol. 2431060554

22. Imanaka H, Taenaka N, Nakamura J, Aoyama K, Hosotani H. Ocular surface disorders in the critically ill. Anaesthesia and Analgesia. 1997;85(2):343-7. DOI: https://doi.org/10.1213/00000539-199708000-00018

23. Christine AS, Theresa AM, Evelyn MK, Jean C, Pat C, Debra R. et al. Am J Crit Care. 2011;20(1):26-34. DOI: https://doi.org/ $10.4037 /$ ajec2011754

24. Anna-Barbara S, Ruud JH, Joseph GAS. Paediatric Pressure Ulcer Prevalence: A Multicenter, Cross-Sectional, Point Prevalence Study in Switzerland. Ostomy wound manag. 2012;18-31.

25. Gershan LA, Esterly NB. Scarring alopecia in neonates as a consequence of hypoxaemia-hypoperfusion. Arch Dis Child 1993;68(5):591-3. DOI: https://doi.org/10.1136/adc.68.5_Spec_No.591

26. Marty V, Alice K, Ann MN, Pat S, Teresa T, David P, et al. A Quality-Improvement Collaborative Project to Reduce Pressure Ulcers in PICUs. Paediatrics. 2013;131(6):1950-60. DOI: https://doi.org/10.1542/peds.2012-1626 